\title{
Modelo de calidad en uso para portales web bancarios en el Perú
}

\section{Quality model in use for bank web portals in Peru}

\author{
Fany Sobero ${ }^{1, a}$, Lenis Wong ${ }^{1, b}$ \\ ${ }^{1}$ Universidad Nacional Mayor de San Marcos, Facultad de Ingeniería de Sistemas e Informática, Unidad de Posgrado. Lima, Perú \\ aEmail: fsoberor@unmsm.edu.pe \\ bEmail: lwongp@unmsm.edu.pe
}

\section{Resumen}

La banca electrónica surgió ante el aumento de la demanda de los clientes como un canal alternativo de fácil acceso, que permite el ahorro de tiempo en su desplazamiento físico, siendo importante que el cliente sienta confianza y que este canal satisface su necesidad. Las aplicaciones bancarias pueden generar riesgos latentes si no se han implementado adecuadamente con ello se genera una mala percepción de los servicios de banco en general. Por ello, en la literatura existen diversos estudios sobre la aplicación de modelos de calidad en diversos rubros, pero no se han aplicado a la banca electrónica peruana, por esta razón, en el presente trabajo se propone un modelo de calidad en uso para portables web bancarias en Perú que permita conocer la percepción al usar el canal electrónico. El modelo consta de 6 características y 11 sub características, lo cual fueron obtenidas por la valoración de expertos en la banca electrónica. Para corroborar la validez de los datos se aplicó alfa de Crombach obteniéndose un $85 \%$ de confiabilidad de los datos. Como principal resultado se obtuvo que la sub característica "seguridad" se muestra como la más importante siendo este factor el más sensible como determinante de la adopción de los canales electrónicos, seguido de la "eficiencia" y "efectividad".

Palabras clave: Historia calidad en uso; portales web; banca electrónica.

\begin{abstract}
Electronic banking emerged as a result of the increase in customer demand as an easily accessible alternative channel, which saves time on their physical travel, and it is important that the customer feel confident and that this channel meets their needs. Banking applications can generate latent risks if they have not been properly implemented, thus generating a bad perception of bank services in general. For this reason, in the literature there are various studies on the application of quality models in various areas, but they have not been applied to Peruvian electronic banking, for this reason, in this paper, we propose a quality model in use for portable web banking. In Peru that allows to know the perception when using the electronic channel. The model consists of 6 characteristics and 11 sub characteristics, which were obtained by the assessment of experts in electronic banking. To corroborate the validity of the data, Crombach's alpha was applied, obtaining $85 \%$ reliability of the data. As a main result, it was obtained that the sub-characteristic "security" is shown as the most important, this factor being the most sensitive as a determinant of the adoption of electronic channels, followed by "efficiency" and "effectiveness".
\end{abstract}

Keywords: Quality in use; web portals; electronic banking.

Sobero, F. \& Wong, L. (2020) Modelo de calidad en uso para portales web bancarios en el Perú. Revista Peruana de Computación y Sistemas, 3(1):3-13. http://dx.doi.org/10.15381/rpcs.v3i1.18349

(c) Los autores. Este artículo es publicado por la Revista Peruana de Computación y Sistemas de la Facultad de Ingeniería de Sistemas e Informáticade la Universidad Nacional Mayor de San Marcos. Este es un artículo de acceso abierto, distribuido bajo los términos de la licencia Creative Commons Atribucion - No Comercia_Compartir Igual 4.0 Internacional. (http://creativecommons.org/licenses/by-nc-sa/4.0/) que permite el uso no comercial, distribución y reproducción en cualquier medio, siempre que la obra original sea debidamente citada. 


\section{Introducción}

En un modelo tradicional las instituciones financieras miden su participación en el mercado por el número de sucursales aperturadas, teniendo en cuenta la necesidad de cercanía física de estas instituciones y de brindar atención al creciente número de clientes que acceden a los servicios financieros. Esto involucra el incremento de costos operativos que hacen poco sustentable este modelo, ante esto, se introduce los canales de atención alternativos que impulsan la banca electrónica, con lo cual disminuye tiempo de atención y costos operativos.

La banca electrónica surgió ante el aumento de la demanda de los clientes siendo un instrumento imprescindible en los servicios financieros, en vista que facilitan el acceso a tecnologías de pago mediante tarjetas de crédito, tarjetas de débito, transferencias electrónicas y entre otros mecanismos [1]. Las plataformas de banca electrónica permiten a los clientes tener fácil acceso a toda la información sobre sus operaciones de crédito y las ofertas de crédito disponibles con la información relevante y algunas de las transacciones de préstamos desde sus localidades [2] .La Banca electrónica es un canal alternativo de fácil acceso, que permite el ahorro de tiempo considerando también que muchas de las operaciones realizadas tienen costos bajos o algunos casos es gratuito.

Las aplicaciones bancarias puestas a disposición al público usuario, a través de los portales web, son consideradas aplicaciones sensibles al ser blanco de ataques para la obtención de los datos personales, bancarios y por las transacciones monetarias que se realizan a través de ellas. Cuando el usuario decide no usarlas por desconfianza o por considerar un proceso que no satisface su necesidad opta por el transporte o custodia de altas sumas de dinero, lo cual produce el incremento de asaltos o pérdidas al quedar desprotegidos ante acontecimientos delictivos o desastres naturales, siendo a su vez una barrera para la promoción y desarrollo [1].

Las aplicaciones bancarias pueden generar riesgos latentes si no se han implementado adecuadamente. Fallas en sistemas de banca electrónica, conexión con la red o sistemas obsoletos pueden causar inconvenientes con los clientes y/o rechazo por parte de estos que al no ver sus expectativas satisfechas, con ello se genera una mala percepción de los servicios de banco en general, viéndose afectado su reputación [3]. Ante esto considerar brindar un servicio de calidad a través de un producto software deberá reflejarse en la efectividad para el cliente y la rentabilidad para la institución.

La calidad del software es el conjunto de cualidades medibles que determina su utilidad y existencia. El desarrollo del producto debe considerar la confiabilidad, mantenibilidad y flexibilidad para disminuir los costos de mantenimiento y mejora durante el tiempo de utilización y durante las etapas del ciclo de vida del software [4].

Según la Guía técnica sobre evaluación de Software para la Administración Pública "La calidad del producto de software puede ser evaluada midiendo atributos internos (medidas típicamente estáticas de productos intermedios), o midiendo atributos externos (midiendo típicamente el comportamiento del código cuando es ejecutado), o bien midiendo los atributos de aplicación de calidad en uso" [5].

La Calidad en Uso es la perspectiva del usuario en relación a la calidad del producto software cuando éste es usado en un ambiente específico y en un contexto de uso específico. Esta permite conocer en qué medida los usuarios pueden conseguir sus metas en un ambiente particular, en vez de medir las propiedades del software en sí mismo. El modelo de calidad en uso puede ser utilizado para especificar y evaluar los requisitos de la calidad en uso de cualquier aspecto del sistema, incluidos el efecto de la calidad del software en un contexto de uso determinado [4].

Según estudio realizado por OEA en América Latina y Caribe, se pudo notar que los usuarios prefieren los medios virtuales en comparación con los presenciales, lo cual requiere un alto nivel de digitalización de los servicios prestados por las instituciones bancarias y tal como lo muestra la Fig.1 las operaciones a través de internet usando computadores es la que registra mayor número de operaciones [6].

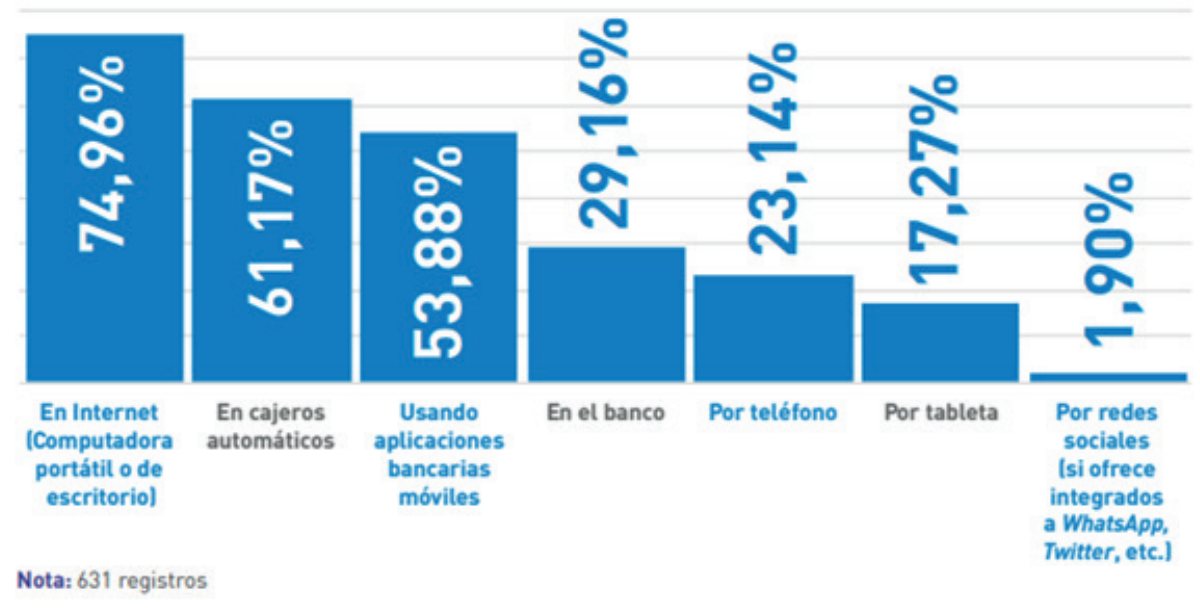

Fig. 1. Medios para revisar transacciones recientes y saldos disponibles [6] 
La revisión de la literatura que se ha realizado en el presente trabajo es sobre diferentes modelos de calidad en uso aplicados en diversos rubros, tales como: aplicación de un modelo de calidad en uso a un portal informativo en línea sobre la salud en Malasia [7], evaluación de aplicaciones web 2.0 [8], medición de experiencias de usuarios de un portal web para la busca de empleos [9], entre otras. Pero estos modelos no se han aplicado a la banca electrónica peruana y sabiendo la importancia de las aplicaciones bancarias hoy en día en el Perú y la vulnerabilidad que estas sufren [3], es por esa razón que el presente trabajo propone un modelo de calidad en uso con la finalidad de conocer la utilidad percibida por los usuarios de los bancos peruanos y de esta manera las instituciones puedan aumentar la efectividad para el cliente y su rentabilidad.

El presente trabajo está organizado de la siguiente manera. En la sección 2, se presentan los trabajos respecto a la literatura relacionada a modelos de calidad en uso, en la sección 3, se presenta el procedimiento a seguir para la obtención del modelo de calidad en uso, en la sección 4, se presenta la metodología de investigación, los resultados y la validación del estudio. Por último, en la sección 5 se encuentran las conclusiones y trabajos futuros respecto a lo desarrollado.

\section{Revisión de la literatura}

Diversas propuestas sobre modelos de calidad en uso han surgido en la literatura, a continuación se presentan algunas investigaciones.

En la propuesta de Flogi y Guida [10], analizan la calidad del sitio web identificando cuatro conceptos (calidad final, en uso, básica e interna) y definiendo las relaciones que tienen con los grupos de interés, centrándose en la calidad en uso y planteando un nuevo modelo de calidad. Como principal aporte aplican la evaluación a través de evaluadores expertos en lugar de usuarios, con lo cual se reduce el tiempo y complejidad de la evaluación.

Alfonzo e Itatí [11], presentan un índice de evaluación web para medir, evaluar y analizar el grado de cumplimiento de sitios web bancarios en Argentina, teniendo en cuenta a clientes potenciales y propios, lo aplican únicamente a dos bancos.

Cordova et. al. [12] propone un modelo de calidad para portales bancarios el cual se denomina BPQM donde en el proceso de evaluación obtiene que la cantidad y calidad de servicios afecta a la satisfacción de los usuarios así también los temas de seguridad, entrenamiento y usabilidad tienen impacto en la calidad de portales bancarios.

En la propuesta de Hussain y Mkpojiogu [7], aplican el modelo de calidad en uso definido en ISO/IEC 25010 a un portal informativo en línea sobre la salud en Malasia, obteniéndose como resultado que el sistema e-Ebola tuvo un nivel bueno en calidad en uso midiéndose la eficacia, eficiencia, satisfacción, seguridad en uso que sería un equivalente a la característica de libertad de riesgo y flexibilidad que se encuentra dentro de la característica cobertura de contexto.

Por otro lado, Orehovacki et. al. [8], plantean un modelo de calidad en uso para aplicaciones web 2.0 compuesto por seis características y 33 sub características para la evaluación se usaron los métodos de registro de uso, cuestionario y pensamiento retrospectivo en voz alta. Analizaron el conjunto de atributos de calidad en uso que deben medirse de forma independiente del tipo de aplicación Web 2.0 y los autores concluyeron que la importancia de un atributo y categoría depende del tipo de la evaluación de la Aplicación Web 2.0 y existe el conjunto de atributos dirigido a medir la calidad en el uso de tipos específicos de aplicaciones Web 2.0.

La ISO/IEC 25010 [13] Plantea un modelo de calidad en uso con cinco características las cuales son efectividad, eficiencia, satisfacción, libertad de riesgo y cobertura del contexto las cuales agrupan a $11 \mathrm{sub}$ características.

Finalmente, Ashok et. al. [9], Usan el modelo de ISO/IEC 25010 con el objetivo de medir la experiencia de usuario que serían los ciudadanos en Malasia que utilizan la página de busca de empleo provista por el gobierno, el alcance abarca la calidad en uso y producto, obteniéndose como resultado que la complejidad de la página por la cantidad de datos requeridos impacta en la obtención de los indicadores y presenta poca adaptabilidad al dispositivos móviles que hoy en día es muy importante para los usuarios.

\section{Procedimiento para obtención del modelo propuesto}

Para la definición del modelo se siguió el procedimiento mostrado en la Fig. 2 el cual constó de siete pasos desarrollados.

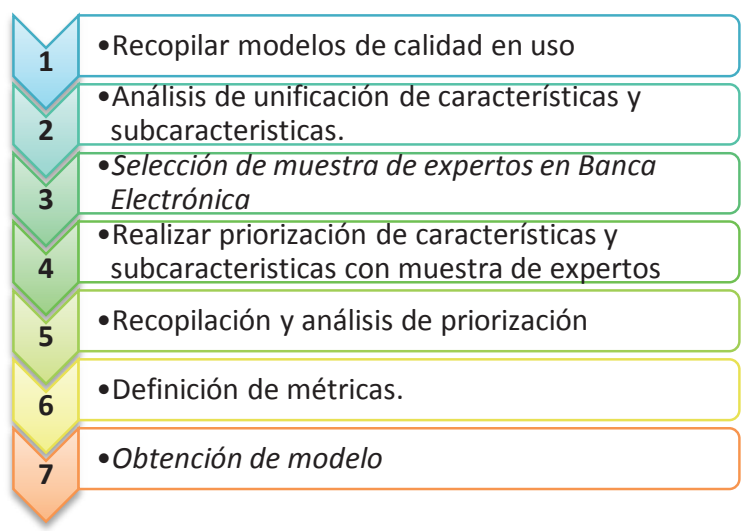

Fig. 2. Procedimiento para la definición del modelo de calidad en uso para portales web bancarios.

\subsection{Recopilar modelos de calidad en uso}

Mediante la revisión de literatura se procedió a recopilar modelos de calidad en uso de diversos estudios realizados los cuales plantean un conjunto de 
características y sub características que conjuntamente con la norma ISO/IEC 25010, en total se consideró siete modelos $[12,10,7,18,11,9,13]$.

\subsection{Análisis de unificación de características y sub características}

Se recopiló los modelos de calidad en uso del paso anterior con los cuales se procedió a realizar el análisis de lo que cada autor consideró en cada sub característica y sus relaciones con las característica a fin se pueda realizar una conciliación que permita obtener un conjunto diferenciado que será considerado como línea base de modelo a plantear.

\subsection{Selección de muestra de expertos en Banca Electrónica.}

Para poder plantear el modelo se requirió de la participación de expertos de Tecnología de la Información en banca electrónica los cuales contaban con un mínimo de 5 años de experiencia, esto a fin de garantizar el conocimiento del canal y que pudieran reflejar su percepción de calidad en uso esperada. Estos profesionales formaban parte del staff de entidades Financiera o personal perteneciente a consultoras de Software, los cuales fueron seleccionados de manera no probabilística mediante la técnica de muestreo denominada bola de nieve.

\subsection{Realizar priorización de características y sub características con muestra de expertos}

Definida la muestra de expertos en el paso anterior se procedió a remitir mediante correo electrónico, en archivos Excel, el instrumento que permitió recabar la opinión de los expertos con respecto al nivel de importancia que le asignan a las sub características relacionadas a la calidad en uso.

Se aplicó la técnica de selección Binaria [14], la cual constó de los siguientes pasos:

- Se listó sub características unificadas.

- Se realizó un glosario de términos la descripción de las sub características,

- Con los miembros de la muestra de expertos se procedió a registrar una matriz en la cual puntuaron cada sub característica considerando la importancia que estas tienen en relación a la calidad en uso para portales web bancarios.

\subsection{Recopilación y análisis de priorización}

Recabados los archivos, mediante correo electrónico, remitidos por los expertos se procedió a verificar las mismas, constatando que estén completas de acuerdo al formato y siguiendo las instrucciones establecidas. Las encuestas que fueron consideradas válidas permitieron la recopilación del score que es calculado en cada encuesta, el cual indica en nivel de importancia o priorización que cada experto. El conjunto de valores recopilados fueron promediados para obtener un valor referencial para cada sub característica para luego proceder a ordenarlas de mayor a menor valor.

\subsection{Obtención de modelo}

Aquellas sub características que tuvieron asociados valores promedio más altos, fueron consideradas como parte del modelo definido. Cada sub característica se relacionó con una o más características.

\subsection{Definición de métricas}

Para cada sub característica que forma parte del modelo planteado se procedió a asociarle una o más métricas que permitirán una medición cuantitativa del grado de cumplimiento alcanzado para cada sub característica.

\section{Validación y Resultados}

Para el desarrollo del presente trabajo se aplicó la siguiente metodología de investigación:

\subsection{Tipo y diseño de investigación}

La presente investigación es del tipo no experimental en vista que se realiza el estudio sin manipulación del contexto. El diseño es de tipo descriptivo pues permitirá conocer la opinión de expertos en banca electrónica como perfil específico de estudio para definir el modelo de calidad en uso [15]. Y se considera como unidad de análisis el canal de banca electrónica de los bancos en el Perú, específicamente el canal de Banca por internet.

\subsection{Población y muestra}

La población del estudio fueron los profesionales de tecnología de la información que laboran en entidades Financieras en áreas de Banca Electrónica, en la actualidad el Perú, cuenta con 61 entidades financieras reconocidas por el Banco Central de Reserva del Perú [16].

En vista que la muestra a seleccionar está conformado por profesionales de tecnología de la información que laboran en entidades Financieras en áreas de Banca Electrónica, no se conoce con exactitud cuántos profesionales conforman la población exactamente, por tanto se aplicó la ecuación (1) para el cálculo de muestra en la cual no se conoce el tamaño de la población.

$$
n=\left(Z_{a}^{2} \times p \times q\right) / d^{2}
$$

Donde:

$\mathrm{Z}=$ nivel de confianza

$\mathrm{p}=$ probabilidad esperada

$\mathrm{q}=$ probabilidad de fracaso

$\mathrm{d}=$ precisión

Ver (2) donde se aplica la fórmula

$$
n=\frac{(1.96)^{2} \times 0.85 \times 0.15}{(0.2)^{2}}
$$


Se obtiene el valor de n igual a 12.26 representando para el estudio total de 12 encuestas a realizar.

La selección de la muestra se realizó mediante la técnica no probabilística denominada bola de nieve, mediante la cual se contactó a profesionales de TI que cuenten con experiencia mínima de 5 años en banca electrónica, es decir, que trabajen o hayan trabajado con aplicativos de este canal, solicitándole a los mismos nos permitan contactar a otros profesionales del área que podrían ser colegas de los mismos que quisieran apoyar en el presente estudio.

Este tipo de muestro permitió tener mayor probabilidad de contar con una muestra con características similares en cuanto a su experiencia profesional [4].

\subsection{Técnica de recolección de datos}

Como técnica que permitió recolectar datos se usó la Evaluación Sistemática Binaria [14] mediante la cual se solicitó a los participantes indicar que sub características de calidad en uso para portales web bancarios considera son importantes de medir que permitan determinar el nivel de calidad en uso que los usuarios perciben y luego se procederá a solicitar que mediante el análisis de pares calcular la prioridad asignada a las sub características seleccionadas.

\subsection{Obtención del modelo}

Para la obtención del modelo se aplicó el procedimiento definido en el punto 3 , tal como se muestra a continuación.

\subsubsection{Recopilar modelos de calidad en uso}

Mediante la revisión de la literatura sobre modelos de calidad en uso se recopiló siete modelos como se muestra en la Tabla 1, los cuales conforman la información base, seis modelos corresponden a estudios realizados en diversos ámbitos y se considera también el modelo definido por la ISO/IEC 25010 correspondiente a la calidad en uso.

4.4.2. Análisis de unificación de características y sub características

Para poder realizar la unificación de características y sub características de los modelos seleccionados se procede a realizar un mapeo general con el cual se procederá al análisis y trazabilidad en vista que la nomenclatura utilizada por cada autor puede ser diversa pero

Tabla 1. Recopilación de modelos de calidad en uso.

\begin{tabular}{|c|c|c|c|c|c|c|c|}
\hline Sub características & [8] & [12] & [11] & [10] & [7] & [9] & [13] \\
\hline Calidad del sistema & $x$ & & & & & & \\
\hline Calidad de servicio & $x$ & & & & & & \\
\hline Calidad de contenido & $x$ & & & & & & \\
\hline Actuación & $x$ & & & & & & \\
\hline Esfuerzo & $x$ & & & & & & \\
\hline Aceptabilidad & & $x$ & & & & & \\
\hline Empatía & & $x$ & & & & & \\
\hline Usabilidad & & $x$ & & $x$ & $x$ & & \\
\hline Seguridad & & $x$ & & & $x$ & & \\
\hline Funcionalidad & & $x$ & & & & & \\
\hline Visibilidad & & & $\mathrm{x}$ & & & & \\
\hline Navegabilidad & & & $x$ & & & & \\
\hline Accesibilidad & & & $x$ & $x$ & & & \\
\hline Calidad de contenido & & & $x$ & & & & \\
\hline Impacto & & & & $x$ & & & \\
\hline Utilidad & & & & $x$ & & & \\
\hline Flexibilidad de uso & & & & & $x$ & & \\
\hline Eficacia & & & & & $x$ & $x$ & \\
\hline Eficiencia & & $x$ & & & & $x$ & $x$ \\
\hline Aprendibilidad & & & & & & $x$ & \\
\hline Operabilidad & & & & & & $x$ & \\
\hline Protección contra errores del usuario & & & & & & $x$ & \\
\hline Libertad de riesgo & & & & & & $x$ & \\
\hline Integridad del contexto & & & & & & $x$ & \\
\hline Efectividad & & & & & & & $x$ \\
\hline Satisfacción & & & & & & & $x$ \\
\hline Libertad de riesgo & & & & & & & $x$ \\
\hline Cobertura de contexto & & & & & & & $x$ \\
\hline
\end{tabular}


puede representar un mismo concepto de medición en el ámbito de la calidad en uso. Para ello se procede a identificar el concepto que cada autor plasma en cada sub característica y se realiza comparaciones con otros similares de tal manera que solo se pueda consolidar conceptos, del total de las sub características recopiladas se obtuvo un total de 21 sub características.

4.4.3. Selección de muestra de expertos en Banca Electrónica

En este paso corresponde la selección de la muestra por lo que se aplicó la selección de muestra no probabilística denominada bola de nieve, solicitando a expertos en TI, específicamente de banca electrónica, que faciliten el contacto con otros expertos en la materia para que puedan realizar la encuesta que permita refinar el modelo de calidad en uso. Se logró contactar a 16 expertos a los cuales se les envió el instrumento, obteniendo la respuesta de 14 y siendo consideradas 12 encuestas válidas por haber sido completamente llenadas y con datos correctos.

4.4.4. Realizar priorización de características y sub características con muestra de expertos

Como primer paso se adaptó los formatos que forman parte de la evaluación sistemática Binaria [14] que permitirá definir las características y sub características del modelo. Para ello se seleccionó las características de calidad en uso de la revisión de literatura y se solicitó a los participantes convocados a indicar si consideran relevantes estas sub características indicando " 1 " o "0" de caso contrario. El Apéndice A muestra el formato que fue usado, en el cual se muestran las sub características listadas que son un total de 21 y se describe cada una de ellas como ayuda al entendimiento de concepto.

Habiendo indicado lo que para los participantes en el estudio consideran son las características más importantes aplicadas a la calidad en uso se procede a realizar la priorización de las mismas en un análisis de pares, para ello se muestra en Tabla 2 el formato usado, en él se solicita se identifique cuál de las sub características es la más importante en cada par, si el atributo del lado izquierdo es más importante se ingresa 1(uno) de lo contrario 0 (cero).

\subsubsection{Recopilación y análisis de priorización}

De las 12 encuestas válidas recopiladas se obtuvo como resultado la información mostrada en Tabla 3 donde se puede apreciar en primera instancia el resultado de lo que los participantes consideraron son las principales características aplicables a la calidad en uso

Tabla 2. Priorización de las características de calidad en uso

\begin{tabular}{|c|c|c|c|c|c|c|c|c|c|c|c|c|c|c|c|c|c|c|c|c|c|c|c|}
\hline Sub característica & $1 / 0$ & Score & 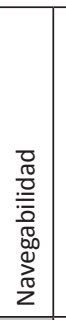 & 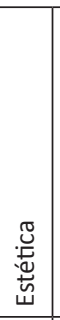 & 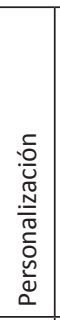 & 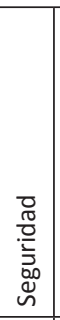 & 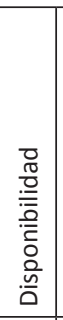 & 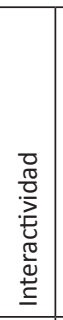 & 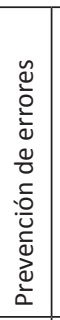 & 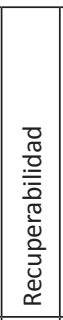 & 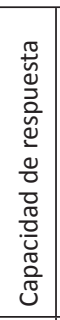 & 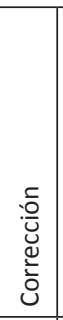 & 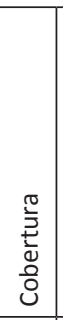 & 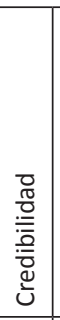 & 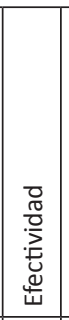 & 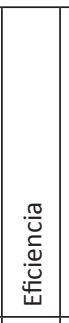 & 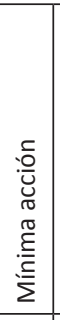 & 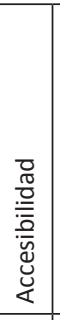 & $\begin{array}{l}0 \\
\frac{0}{3} \\
\frac{1}{0} \\
\frac{0}{0} \\
\frac{\pi}{0} \\
\frac{0}{\overline{0}} \\
\overline{\tilde{\sigma}} \\
\end{array}$ & $\begin{array}{l}\frac{0}{\pi} \\
\frac{\pi}{\pi} \\
\frac{\pi}{0} \\
\frac{0}{0} \\
\pi \\
\frac{0}{0} \\
0 \\
\frac{\pi}{0} \\
\frac{0}{0} \\
0 \\
0 \\
\end{array}$ & 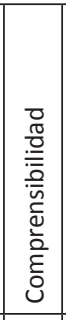 & 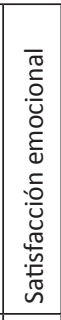 & 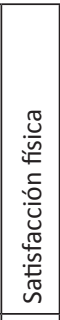 \\
\hline Navegabilidad & 0 & 0 & & & & & & & & & & & & & & & & & & & & & \\
\hline Estética & 0 & 0 & & & & & & & & & & & & & & & & & & & & & \\
\hline Personalización & 0 & 0 & & & & & & & & & & & & & & & & & & & & & \\
\hline Seguridad & 0 & 0 & & & & & & & & & & & & & & & & & & & & & \\
\hline Disponibilidad & 0 & 0 & & & & & & & & & & & & & & & & & & & & & \\
\hline Interactividad & 0 & 0 & & & & & & & & & & & & & & & & & & & & & \\
\hline Prevención de errores & 0 & 0 & & & & & & & & & & & & & & & & & & & & & \\
\hline Recuperabilidad & 0 & 0 & & & & & & & & & & & & & & & & & & & & & \\
\hline Capacidad de respuesta & 0 & 0 & & & & & & & & & & & & & & & & & & & & & \\
\hline Corrección & 0 & 0 & & & & & & & & & & & & & & & & & & & & & \\
\hline Cobertura & 0 & 0 & & & & & & & & & & & & & & & & & & & & & \\
\hline Credibilidad & 0 & 0 & & & & & & & & & & & & & & & & & & & & & \\
\hline Efectividad & 0 & 0 & & & & & & & & & & & & & & & & & & & & & \\
\hline Eficiencia & 0 & 0 & & & & & & & & & & & & & & & & & & & & & \\
\hline Mínima acción & 0 & 0 & & & & & & & & & & & & & & & & & & & & & \\
\hline Accesibilidad & 0 & 0 & & & & & & & & & & & & & & & & & & & & & \\
\hline Facilidad de uso & 0 & 0 & & & & & & & & & & & & & & & & & & & & & \\
\hline Capacidad de aprendizaje & 0 & 0 & & & & & & & & & & & & & & & & & & & & & \\
\hline Comprensibilidad & 0 & 0 & & & & & & & & & & & & & & & & & & & & & \\
\hline Satisfacción emocional & 0 & 0 & & & & & & & & & & & & & & & & & & & & & \\
\hline Satisfacción física & 0 & 0 & & & & & & & & & & & & & & & & & & & & & \\
\hline
\end{tabular}


Tabla 3. Recopilación de datos - Identificación de características

\begin{tabular}{cccccccccccccc} 
& $E 1$ & $E 2$ & $E 3$ & $E 4$ & $E 5$ & $E 6$ & $E 7$ & $E 8$ & $E 9$ & $E 10$ & E 11 & E 12 & Promedio \\
\hline 1 & 8 & 13 & 4 & 8 & 8 & 7 & 5 & 8 & 2 & 11 & 8 & 7 & 7.4 \\
\hline 2 & 11 & 7 & 1 & 0 & 0 & 2 & 0 & 0 & 0 & 3 & 5 & 4 & 2.8 \\
\hline 3 & 7 & 12 & 3 & 7 & 5 & 8 & 0 & 8 & 0 & 3 & 7 & 5 & 5.4 \\
\hline 4 & 6 & 9 & 17 & 15 & 18 & 5 & 8 & 17 & 13 & 19 & 9 & 15 & 12.6 \\
\hline 5 & 8 & 14 & 8 & 2 & 9 & 4 & 6 & 8 & 6 & 17 & 8 & 7 & 8.1 \\
\hline 6 & 5 & 14 & 6 & 0 & 3 & 5 & 0 & 0 & 0 & 9 & 3 & 0 & 3.8 \\
\hline 7 & 7 & 13 & 16 & 0 & 7 & 6 & 3 & 13 & 3 & 10 & 7 & 8 & 7.8 \\
\hline 8 & 4 & 5 & 7 & 11 & 5 & 8 & 0 & 5 & 7 & 4 & 5 & 7 & 5.7 \\
\hline 9 & 8 & 12 & 11 & 2 & 16 & 3 & 4 & 10 & 4 & 13 & 9 & 8 & 8.3 \\
\hline 10 & 9 & 12 & 19 & 9 & 3 & 8 & 3 & 8 & 5 & 11 & 7 & 10 & 8.7 \\
\hline 11 & 7 & 0 & 13 & 5 & 12 & 3 & 0 & 4 & 11 & 2 & 6 & 5 & 5.7 \\
\hline 12 & 0 & 8 & 7 & 7 & 5 & 6 & 7 & 5 & 9 & 5 & 4 & 6 & 5.8 \\
\hline 13 & 0 & 9 & 17 & 11 & 14 & 10 & 6 & 15 & 12 & 16 & 7 & 11 & 10.7 \\
\hline 14 & 0 & 11 & 15 & 12 & 15 & 12 & 3 & 16 & 10 & 18 & 12 & 10 & 11.2 \\
\hline 15 & 7 & 8 & 3 & 2 & 5 & 1 & 0 & 2 & 4 & 9 & 2 & 5 & 4 \\
\hline 16 & 11 & 6 & 5 & 0 & 0 & 0 & 0 & 0 & 8 & 0 & 0 & 2 & 2.7 \\
\hline 17 & 13 & 5 & 7 & 3 & 12 & 9 & 2 & 8 & 11 & 12 & 6 & 10 & 8.2 \\
\hline 18 & 10 & 3 & 8 & 5 & 11 & 0 & 0 & 10 & 8 & 5 & 6 & 7 & 6.1 \\
\hline 19 & 6 & 6 & 6 & 6 & 7 & 6 & 2 & 4 & 8 & 7 & 5 & 7 & 5.8 \\
\hline 20 & 10 & 3 & 6 & 3 & 10 & 11 & 10 & 5 & 5 & 4 & 7 & 6 & 6.7 \\
\hline 21 & 7 & 7 & 10 & 0 & 11 & 0 & 0 & 0 & 0 & 2 & 0 & 0 & 3.1 \\
\hline
\end{tabular}

de la banca por internet. Las características seguridad, eficiencia y efectividad alcanzan los más alto puntajes como sub características menos relevantes satisfacción física, accesibilidad y estética.

En la ecuación (3) se muestra como calcular el coeficiente alfa de Crombach con los datos obtenidos a fin de obtener una medida de la fiabilidad de la escala usada este valor deberá estar comprendido entre 0 y 1 lo cual permitirá conocer la cantidad de error de medición en la prueba efectuada [17].

$$
\alpha=\frac{K}{K-1}\left(1-\frac{\sum_{i=1}^{k} S_{i}}{S_{t}}\right)
$$

Donde:

$\mathrm{K}=$ Número de ítems

$\mathrm{Si}=$ Varianza de cada ítem

St $=$ Varianza de las suma de ítems

En la ecuación (4) se muestra el coeficiente alfa de Crombach obtenido siendo de 0.86 , lo cual indica que los datos derivados del instrumento aplicado ofrecen un buen grado de confiabilidad.

$$
\alpha=\frac{12}{12-1}\left(1-\frac{213.71}{123.51}\right)=0.86
$$

Siendo los datos confiables se procede luego a ordenar los promedios obtenidos de mayor a menor como se muestra en la Tabla 4, de tal manera se pueda evidenciar las características que han alcanzado los mayores promedios.
Tabla 4. Recopilación de datos - Priorización de Características

\begin{tabular}{clc}
$\mathbf{N}^{\circ}$ & Atributo & Promedio \\
\hline 4 & Seguridad & 12.6 \\
\hline 14 & Eficiencia & 11.2 \\
\hline 13 & Efectividad & 10.7 \\
\hline 10 & Corrección & 8.7 \\
\hline 9 & Capacidad de respuesta & 8.3 \\
\hline 5 & Facilidad de uso & 8.1 \\
\hline 17 & Disponibilidad & 8.2 \\
\hline 7 & Prevención de errores & 7.8 \\
\hline 1 & Navegabilidad & 7.4 \\
\hline 20 & Satisfacción emocional & 6.7 \\
\hline 18 & Capacidad de aprendizaje & 6.1 \\
\hline 12 & Credibilidad & 5.8 \\
\hline 19 & Comprensibilidad & 5.8 \\
\hline 11 & Recuperabilidad & 5.7 \\
\hline 8 & Cobertura & 5.7 \\
\hline 3 & Personalización & 5.4 \\
\hline 6 & Mínima acción & 3.8 \\
\hline 15 & Interactividad & 4 \\
\hline 21 & Satisfacción física & 3.1 \\
\hline 16 & Estética & 2.7 \\
\hline 2 & Accesibilidad & 2.8 \\
\hline & &
\end{tabular}

\subsubsection{Obtención del modelo}

Finalmente, la Tabla 5 muestra la selección de las sub características que obtuvieron mayor puntaje, las cuales conformaran el modelo planteado. La Fig. 3 muestra el diagrama del modelo obtenido que consta 


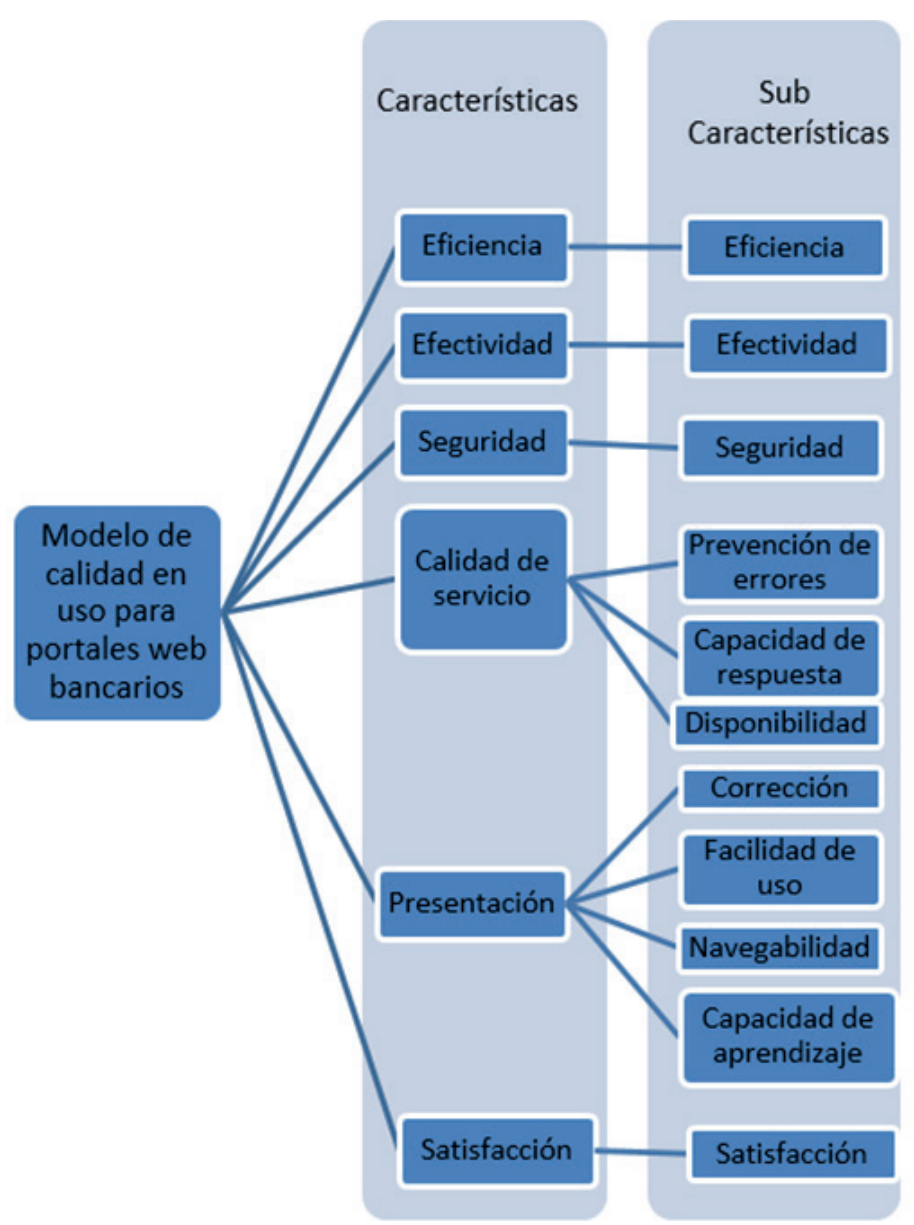

Fig 3. Modelo de calidad en uso para portales web bancarios

de 11 sub características (Eficiencia, Efectividad, Seguridad, Prevención de errores, Capacidad de respuesta, Disponibilidad, Corrección, Facilidad de uso, Navegabilidad, Capacidad de aprendizaje, Satisfacción), estas fueron agrupadas 6 características (Eficiencia, Efectividad, Seguridad, Calidad de servicio, Presentación, Satisfacción) las cuales agrupan sub características que tienen conceptos relacionados pero manteniendo la eficiencia y efectividad guiados por ISO/IEC 25010[13].

Tabla 5. Priorización de Características

\begin{tabular}{clc}
$\mathbf{N}^{\circ}$ & Atributo & Promedio \\
\hline 4 & Seguridad & 12.6 \\
\hline 14 & Eficiencia & 11.2 \\
\hline 13 & Efectividad & 10.7 \\
\hline 10 & Corrección & 8.7 \\
\hline 9 & Capacidad de respuesta & 8.3 \\
\hline 5 & Facilidad de uso & 8.1 \\
\hline 17 & Disponibilidad & 8.2 \\
\hline 7 & Prevención de errores & 7.8 \\
\hline 1 & Navegabilidad & 7.4 \\
\hline 20 & Satisfacción emocional & 6.7 \\
\hline 18 & Capacidad de aprendizaje & 6.1 \\
\hline
\end{tabular}

En la Tabla 6 se describe cada una de las sub características que conforman el modelo indicando lo que se tiene por objetivo medir.

\subsection{Definición de métricas}

En el Apéndice B se muestra las métricas consideradas para medir cada una de las sub características del modelo definido. Con respecto a la efectividad y eficiencia las métricas aplicadas corresponderán a ISO/IEC 25010 y para las demás sub características se plantea métricas que permitan obtener indicadores del cumplimiento del concepto que encierra cada sub característica.

En relación al modelo obtenido se puede observar que la seguridad se muestra como la característica más importante siendo este factor el más sensible como determinante de la adopción de los canales electrónicos, lo cual es mencionado en [6] que indica que al haberse incrementado el uso del entorno digital en entidades bancarias ha producido un conjunto de riesgos, amenazas, vulnerabilidades e incidentes de diversos tipos, a los que han estado expuestos tanto estas organizaciones como sus usuarios llevando a estas entidades a aumentar su presupuesto en seguridad digital en promedio de $54 \%$ debido a nuevas amenazas de ciberseguridad.

\section{Conclusiones}

Es importante el aporte realizado por diversos autores en relación al planteamiento de modelos de calidad en uso para una variedad de aplicaciones, así como, la obtención de mediciones de calidad en uso en base a la 
Tabla 6. Descripción del modelo de calidad en uso para portales web bancarios

\begin{tabular}{lll}
\multicolumn{1}{c}{ Característica } & \multicolumn{1}{c}{ Sub Característica } & \multicolumn{1}{c}{ Descripción } \\
\hline Eficiencia & Eficiencia & Tiempo estimado necesario para completar una tarea especificada \\
\hline Efectividad & Efectividad & Grado en que las tareas se pueden ejecutar con precisión y completamente \\
\hline Seguridad & Seguridad & Contiene funcionalidades y mecanismos que protegen los datos contra accesos no autorizados \\
\hline \multirow{2}{*}{$\begin{array}{l}\text { Calidad de } \\
\text { servicio }\end{array}$} & Prevención de errores & Contiene mecanismo de ayuda para evitar el usuario cometa errores \\
\cline { 2 - 3 } & Capacidad de respuesta & Velocidad de respuesta de la aplicación a las solicitudes y acciones de los usuarios \\
\cline { 2 - 3 } & Disponibilidad & Tiempo que la aplicación web está disponible para el usuario cuando es necesario \\
\hline \multirow{3}{*}{ Presentación } & Corrección & La información es correcta, precisa y válida. \\
\cline { 2 - 3 } & Facilidad de uso & Los usuarios no tienen que consultar los recursos de ayuda en línea o a un experto. \\
\cline { 2 - 3 } & Navegabilidad & Encontrar y acceder de forma rápida y fácil a todas las funcionalidades de interfaz \\
\cline { 2 - 3 } & Capacidad de aprendizaje & Fácil de aprender a usar. \\
\hline Satisfacción & Satisfacción & Cumple con las expectativas del usuario \\
\hline
\end{tabular}

norma ISO/IEC 25010 pues reflejan el punto de vista del usuario como parte de la retroalimentación que debe existir para la mejora continua de los servicios de TI. El procedimiento definido para la obtención del modelo de calidad en uso, que consta de 7 pasos fue aplicado permitió obtener la apreciación de los expertos en el sector de banca electrónica que en función a su priorización permitió obtener el modelo planteado que consta de 11 sub características, las cuales se agruparon en 6 características y siendo necesario la definición de la medición de las mismas se definieron las métricas correspondientes.

Es importante considerar que siendo los aplicativos móviles un canal importante en el cual se realizan operaciones bancarias en volumen importante tal como lo indica [6], resulta apreciable contar con modelos de calidad y, de acuerdo con el estudio realizado, modelos de calidad en uso que permitan medir la percepción del grado de calidad que tienen estos aplicativos.

\section{Agradecimientos}

Los autores agradecen a todos a los profesionales de TI en Banca Electrónica que participaron como expertos en el presente estudio, compartiendo su experiencia y su tiempo.

\section{Referencias}

[1] VISA. (2016, 05 04). Informe sobre e-Readiness en Latinoamérica 2014. Retrieved from http://promociones.visa. com/lac/ecommerce/assets/reports/es/peru-ereadiness-report-2014-es.pdf

[2] ASOCIACIÓN LATINOAMERICANA DE INSTITUCIONES FINANCIERAS. (2015). Desarrollo y la creación de productos para la inclusión financiera. Retrieved from http://www19. iadb.org/intal/intalcdi/PE/2015/15195.pdf

[3] Superintendencia de Banca y Seguros. (2000). BANCA ELECTRÓNICA: POSIBILIDADES, RIESGOS Y LINEAMIENTOS REGULATORIOS - UNA PRIMERA APROXIMACIÓN. Lima.

[4] Malhotra, N. (2004). Investigación de mercados: un enfoque aplicado. México: Pearson.

[5] Oficina Nacional de Gobierno Electrónico e Informática. (2004). GUÍA TÉCNICA SOBRE EVALUACIÓN DE SOF-
TWARE PARA ADM. PÚBLICA. Retrieved from http://www. ongei.gob.pe/Bancos/Banco_Normas/archivos/Guia-Evaluacion-SW.pdf

[6] OEA. (2018). Estado de la ciberseguridad en el sector Bancario de América Latina y el Caribe.

[7] Hussain, A., \& Mkpojiogu, E. (2015). An Application of the standar in the quality in use assessment of an online health awareness system ISO/IEC 25010. Jurnal Teknologi , 9-13.

[8] Orehovacki, T., Granic, A., \& Kermeka, D. (2013). Evaluating the perceived and estimated quality in use of Web 2.0 applications. The Journal of Systems and Software, 3039-3059.

[9] Ashok, S., Norfarhana, A., Soo Shi, T., Chuan, N., Zulkifle, M., Siti, H., \& Yoong, S. (2014). Measuring Public Value UX-Based on ISO/IEC 25010 Quality Attributes: Case Study on e-Government Website. 3rd International Conference on User Science and Engineering , 56-61.

[10] Fogli, D., \& Guida, G. (2014). A practical approach to the assessment of quality in use ofcorporate web sites. The Journal of Systems and Software 99, 52 - 65.

[11] Alfonzo, P., \& Itatí Mariño, S. (2013). Propuesta de un indice de evaluación web para la estimación de la calidad de sitios web bancarios. Gerenc. Tecnol. Inform., 15-32.

[12] Córdoba, J., Cachero, C., Calero, C., \& Genero, M. (2007). Modelo de Calidad para portales bancarios. XXXIII Conferencia Latinoamericana de Informática (CLEl'07). San Jose Costa Rica.

[13] ISO. (2020, 01 30). ISO ORG. Retrieved from https://www. iso.org/obp/ui/\#iso:std:iso-iec:25010:ed-1:v1:en

[14] Brosseau, J. (2010). Clarrus - Software Quality Attributes: Following All the Steps. Retrieved 01 2017, from http://www. clarrus.com/

[15] Vara-Horna, A. (2012). Desde La Idea hasta la sustentación: Siete pasos para una tesis exitosa. Lima: Instituto de Investigación USMP.

[16] Banco Central de Reserva. (2020, 03 12). Banco Central de Reserva. Retrieved from https://www.bcrp.gob.pe/sitios-de-interes/entidades-financieras.html

[17] NCBI. (2020, 03 02). Making sense of Cronbach's alpha. Retrieved from https://www.ncbi.nlm.nih.gov/pmc/articles/ PMC4205511/Calero Muñoz, C., Piattini Velthuis, M. G., \& Moraga de la Rubia, M. Á. (2010). Calidad del producto y proceso software. Ra-Ma. 


\section{APÉNDICE}

Apéndice A. Identificación de las características de calidad en uso

\begin{tabular}{|c|c|c|c|}
\hline \# & Sub características & Observación & $1 / 0$ \\
\hline 1 & Navegabilidad & Encontrar y acceder de forma rápida y fácil a todas las funcionalidades de interfaz & \\
\hline 2 & Estética & Grado de atractivo visual. & \\
\hline 3 & Personalización & Capacidad de satisfacer las necesidades de los usuarios. & \\
\hline 4 & Seguridad & Contiene funcionalidades y mecanismos que protegen los datos contra accesos no autorizados & \\
\hline 5 & Disponibilidad & Tiempo que la aplicación web está disponible para el usuario cuando es necesario & \\
\hline 6 & Interactividad & $\begin{array}{l}\text { Crea la sensación de usar una aplicación de escritorio y contiene funcionalidades que facilitan dife- } \\
\text { rentes tipos de interacción entre usuarios }\end{array}$ & \\
\hline 7 & Prevención de errores & Contiene mecanismo de ayuda para evitar el usuario cometa errores & \\
\hline 8 & Recuperabilidad & Capacidad de recuperarse de errores e interrupciones operacionales & \\
\hline 9 & Capacidad de respuesta & Velocidad de respuesta de la aplicación a las solicitudes y acciones de los usuarios & \\
\hline 10 & Corrección & La información es correcta, precisa y válida. & \\
\hline 11 & Cobertura & Debe contener suficiente cantidad de información y no ser abrumador & \\
\hline 12 & Credibilidad & contenido imparcial, confiable y verificable & \\
\hline 13 & Efectividad & Grado en que las tareas se pueden ejecutar con precisión y completamente & \\
\hline 14 & Eficiencia & Tiempo estimado necesario para completar una tarea especificada & \\
\hline 15 & Mínima acción & Cantidad estimada de pulsaciones de teclas y clics del mouse & \\
\hline 16 & Accesibilidad & Grado en el cual puede ser utilizado por personas con más amplia gama de características y capacidades & \\
\hline 17 & Facilidad de uso & Los usuarios no tienen que consultar los recursos de ayuda en línea o a un experto. & \\
\hline 18 & Capacidad de aprendizaje & Fácil de aprender a usar. & \\
\hline 19 & Comprensibilidad & Las funcionalidades de la interfaz son claras e inequívocas para usuario & \\
\hline 20 & Satisfacción emocional & Cumple con las expectativas del usuario & \\
\hline 21 & Satisfacción física & Cumple con las expectativas del usuario a nivel ergonómico & \\
\hline
\end{tabular}

Apéndices B. Métricas definidas para el modelo

\begin{tabular}{|c|c|c|c|c|}
\hline Característica & Sub característica & Métrica & Fórmula & Valor deseado \\
\hline \multirow{12}{*}{ Efectividad } & \multirow{12}{*}{ Efectividad } & \multirow{4}{*}{$\begin{array}{l}\text { Completitud de } \\
\text { tarea }\end{array}$} & $\mathrm{X}=\mathrm{A} / \mathrm{B}$ & $0<=X<=1$ \\
\hline & & & $A=$ Número de tareas terminadas & El más cercano a 1 , el mejor \\
\hline & & & $B=$ Número total de tareas intentadas & \\
\hline & & & Donde $\mathrm{B}>0$ & \\
\hline & & \multirow{4}{*}{$\begin{array}{l}\text { Efectividad de } \\
\text { tarea }\end{array}$} & $X=A / B$ & $0<=X<=1$ \\
\hline & & & $\mathrm{A}=$ Cantidad de obj. terminados por tarea & El más cercano a 1, el mejor \\
\hline & & & $\mathrm{B}=$ Cantidad de obj. planeados a realizar por tarea & \\
\hline & & & Donde $B>0$ & \\
\hline & & \multirow{4}{*}{$\begin{array}{l}\text { Frecuencia de } \\
\text { error }\end{array}$} & $X=A / B$ & $0<=X<=1$ \\
\hline & & & $A=$ Número de errores cometidos & El más cercano a 1, el mejor \\
\hline & & & $\mathrm{B}=$ Número de tareas & \\
\hline & & & Donde $\mathrm{B}>0$ & \\
\hline
\end{tabular}


Apéndice B. Continuación

\begin{tabular}{|c|c|c|c|c|}
\hline Característica & Sub característica & Métrica & Fórmula & Valor deseado \\
\hline \multirow{12}{*}{ Eficiencia } & \multirow{12}{*}{ Eficiencia } & \multirow{4}{*}{$\begin{array}{l}\text { Tiempo de la } \\
\text { tarea }\end{array}$} & $X=A / B$ & $0<=X<=1$ \\
\hline & & & $A=$ Tiempo actual & $\begin{array}{l}\text { Si } A<=B \text { más cercano a } 0 \text { es } \\
\text { mejor. }\end{array}$ \\
\hline & & & B=Tiempo planeado & \multirow{2}{*}{$\begin{array}{l}\text { Si A>B será considerado el peor } \\
\text { caso. }\end{array}$} \\
\hline & & & Donde $B>0$ & \\
\hline & & \multirow{4}{*}{$\begin{array}{l}\text { Tiempo relativo } \\
\text { de la tarea }\end{array}$} & $X=A / B$ & $0<=X<=1$ \\
\hline & & & $\mathrm{A}=$ Tiempo que termina la tarea un usuario experto & El más cercano a 1, el mejor \\
\hline & & & $\mathrm{B}=$ Tiempo que termina la tarea un usuario normal & \\
\hline & & & Donde $\mathrm{B}>0$ & \\
\hline & & \multirow{4}{*}{$\begin{array}{l}\text { Número } \\
\text { relativo de las } \\
\text { acciones del } \\
\text { usuario }\end{array}$} & $X=A / B$ & $0<=X<=1$ \\
\hline & & & $A=$ Número de acciones realizadas por los usuarios & El más cercano a 1, el mejor \\
\hline & & & $B=$ Número de acciones necesarias & \\
\hline & & & Donde $\mathrm{B}>0$ & \\
\hline Seguridad & Seguridad & $\begin{array}{l}\text { Grado de } \\
\text { percepción de } \\
\text { seguridad }\end{array}$ & $\begin{array}{l}X=A / B \\
A=\text { Número de preguntas con respuesta satisfactorias } \\
B=\text { Número de preguntas del cuestionario } \\
\text { Donde } B>0\end{array}$ & $\begin{array}{l}0<=X<=1 \\
\text { El más cercano a } 1, \text { el mejor }\end{array}$ \\
\hline \multirow{6}{*}{$\begin{array}{l}\text { Calidad de } \\
\text { servicio }\end{array}$} & \multirow{3}{*}{$\begin{array}{l}\text { Prevención de } \\
\text { errores }\end{array}$} & \multirow{3}{*}{$\begin{array}{l}\text { Evitar el error } \\
\text { de operación } \\
\text { del usuario }\end{array}$} & $X=A / B$ & $0<=X<=1$ \\
\hline & & & $\begin{array}{l}\mathrm{A}=\text { Número de acciones de usuario y entradas que son } \\
\text { protegido de causar cualquier mal funcionamiento del } \\
\text { sistema }\end{array}$ & El más cercano a 0 es el mejor. \\
\hline & & & $\begin{array}{l}\text { B = Número de acciones del usuario y entradas que } \\
\text { podría protegerse de causar cualquier sistema mal } \\
\text { funcionamiento }\end{array}$ & \\
\hline & \multirow{3}{*}{$\begin{array}{l}\text { Capacidad de } \\
\text { respuesta }\end{array}$} & \multirow{3}{*}{$\begin{array}{l}\text { Tiempo de } \\
\text { espera }\end{array}$} & $X=B-A$ & \multirow{3}{*}{ El más cercano a 0 es el mejor. } \\
\hline & & & $\mathrm{A}=$ Tiempo cuando se inicia un trabajo & \\
\hline & & & $\mathrm{B}=$ Tiempo en completar el trabajo & \\
\hline \multirow{13}{*}{ Presentación } & \multirow{3}{*}{ Corrección } & \multirow{3}{*}{$\begin{array}{l}\text { Categorización } \\
\text { de información } \\
\text { comprensible }\end{array}$} & $X=A / B$ & $0<=X<=1$ \\
\hline & & & $\begin{array}{l}A=\text { Número de estructuras de información que son } \\
\text { familiares para los usuarios. }\end{array}$ & El más cercano a 1, el mejor \\
\hline & & & B = Número de estructuras de información utilizadas & \\
\hline & \multirow{3}{*}{ Facilidad de uso } & \multirow{3}{*}{$\begin{array}{l}\text { Interfaz de } \\
\text { usuario auto } \\
\text { explicativa }\end{array}$} & $X=A / B$ & $0<=X<=1$ \\
\hline & & & $\begin{array}{l}\text { A = Número de elementos de información y pasos que } \\
\text { son entendibles para el usuario. }\end{array}$ & El más cercano a 1, el mejor \\
\hline & & & $\begin{array}{l}\text { B = Número de elementos de información y pasos nece- } \\
\text { sarios para completar tareas comunes para un usuario } \\
\text { inexperto }\end{array}$ & \\
\hline & \multirow{4}{*}{ Navegabilidad } & \multirow{4}{*}{ Navegabilidad } & $X=A / B$ & $0<=X<=100$ \\
\hline & & & A= Número elementos de navegabilidad & El más cercano a 1, el mejor \\
\hline & & & $\mathrm{B}=$ Número elementos de navegabilidad proyectados & \\
\hline & & & Donde $\mathrm{B}>0$ & \\
\hline & \multirow{3}{*}{$\begin{array}{l}\text { Capacidad de } \\
\text { aprendizaje }\end{array}$} & \multirow{3}{*}{$\begin{array}{l}\text { Interfaz de } \\
\text { usuario auto } \\
\text { explicativa }\end{array}$} & $X=A / B$ & $0<=X<=1$ \\
\hline & & & $\begin{array}{l}\text { A = Número de elementos de información y pasos que } \\
\text { son entendibles para el usuario }\end{array}$ & El más cercano a 1, el mejor \\
\hline & & & $\begin{array}{l}\text { B = Número de elementos de información y pasos nece- } \\
\text { sarios para completar tareas para un usuario inexperto }\end{array}$ & \\
\hline \multirow{4}{*}{ Satisfacción } & \multirow{4}{*}{ Satisfacción } & \multirow{4}{*}{$\begin{array}{l}\text { Nivel de } \\
\text { satisfacción }\end{array}$} & $X=A / B$ & $0<=X<=1$ \\
\hline & & & $A=$ Número de preguntas con respuesta satisfactorias & El más cercano a 1, el mejor \\
\hline & & & B=Número de preguntas del cuestionario & \\
\hline & & & Donde $\mathrm{B}>0$ & \\
\hline
\end{tabular}


